\title{
Neonatal Seizures: Incidence, Etiologies, Clinical Features and EEG Findings in the Neonatal Intensive Care Unit
}

\author{
Su-Ching $\mathrm{Hu}^{1}$, Kun-Long Hung ${ }^{1,2^{*}}$ and Hui-Ju Chen ${ }^{1,3}$ \\ ${ }^{1}$ Department of Pediatrics, Cathay General Hospital, Taipei, Taiwan \\ ${ }^{2}$ School of Medicine, Fu-Jen Catholic University, New Taipei City, Taiwan \\ ${ }^{3}$ Department of Pediatric Neurology, Mackay Children's Hospital, Taipei, Taiwan
}

"Corresponding author: Kun-Long Hung, Department of Pediatrics, Cathay General Hospital, Taipei 280, Jen Ai Road Section 4, Taipei, Taiwan, Tel: 886-2-27082121/1016; Fax: 886-2-27082423; E-mail: klhung@cgh.org.tw

Received date: Dec 19, 2016; Accepted date: Jan 06, 2017; Published date: Jan 10, 2017

Copyright: (C) $2017 \mathrm{Hu} \mathrm{SC}$, et al. This is an open-access article distributed under the terms of the Creative Commons Attribution License, which permits unrestricted use, distribution, and reproduction in any medium, provided the original author and source are credited.

\begin{abstract}
Objective: To evaluate the incidence, etiologies, clinical features and EEG findings of seizures among the neonates admitted to one medical center.

Method: We retrospectively reviewed the medical records of neonates who were diagnosed to have neonatal seizure at the Neonatal Intensive Care Unit (NICU) of one medical center in Taiwan from January to December 2015. The clinical pictures, laboratory data, image and EEG findings were reviewed in all cases.

Results: Neonatal seizures were diagnosed in 22 among 122 neonatal admissions in NICU in 2015. The most common type of seizures observed were subtle $(77.2 \%)$, followed by tonic $(13.7 \%)$ and clonic $(9.1 \%)$ seizures. Acidosis was noted in cases with perinatal asphyxia and shock. The other laboratory data was non-contributory. Seventeen (77.3\%) cases had abnormal EEG (focal spikes in majority) and $12(54.5 \%)$ cases had abnormal image findings. Perinatal asphyxia (59.1\%) was the most common cause of neonatal seizures, followed by CNS infection $(18.2 \%)$, malformation syndrome $(9.1 \%)$ and intracranial hemorrhage $(9.1 \%)$.
\end{abstract}

Conclusion: Neonatal seizures were found in about $18 \%$ of our NICU admissions in one year and the most common was subtle type. Perinatal asphyxia was the most common etiology of neonatal seizures, followed by CNS infection. EEG plays a major role in the diagnosis and follow-up of neonatal seizures.

Keywords: Neonatal seizure; Neonatal Intensive Care Unit (NICU); EEG; Perinatal asphyxia

\section{Introduction}

Seizures are more common in neonatal period than any other life time. The incidence is approximately 3 per 1000 live births. Neonatal seizure can have variable clinical manifestations. According to Volpe's textbook, neonatal seizures can be classified as subtle, myoclonic, clonic or tonic [1]. Subtle seizure can occur in both full-term and preterm baby. Clonic seizure occurs more common in full-term infants. Tonic seizure and myoclonic seizure occur more frequently in preterm than full-term infants. Among these seizure types in neonates, subtle seizures are far more common than other types of neonatal seizures, but more difficult to recognize. Myoclonic seizures are always associated with more severe brain damage and mostly poor in prognosis.

Common causes of neonatal seizure include Hypoxic-Ischemic Encephalopathy (HIE), Central Nervous System (CNS) infections, intracranial hemorrhage, ischemic stroke, malformation, metabolic disorder, specific syndromes, and rarely, primary epilepsy. Regardless of the etiology, neonatal seizures may cause damage to the brain and lead to developmental delay, learning disability, and epilepsy. Intractable seizure and status epilepticus tend to be associated with poor outcome. Here we report a retrospective study of neonatal seizure in the Neonatal Intensive Care Unit (NICU) in one medical center within one year period.

\section{Method}

There were a total of 122 neonates admitted to our NICU from January 1, 2015 to December 31, 2015. We conducted a retrospective review of medical records of 22 neonates who developed seizures during admission. Neonatal seizure was defined as paroxysmal events with at least one of the following clinical characteristics: Changes in behavior, stereotyped or periodic motor activities or autonomic dysfunction [1].

In order to determine the etiology of seizures, complete physical, neurological examination, laboratory tests such as metabolic profiles including blood glucose, lactic acid, ammonia, arterial blood gas analysis and electrolytes such as serum calcium, sodium and magnesium levels were done in all cases. In suspected cases of sepsis, full septic work-up including complete blood counts, blood cultures and CSF examination with culture was done. Neuroimaging (brain sonogram or MRI) and EEG were done in all cases. The amplitude integrated Electroencephalography (aEEG) was monitored in HIE case and those diagnosed with meningitis, sepsis and others. 
Birth history with gestational age, mode of delivery, birth event Apgar score and antenatal problem was recorded. Seizure frequency, pattern, and age of onset were also collected.

\section{Results}

A total number of 22 patients out of 122 NICU admissions were found to have neonatal seizure. Among them, fourteen patients were males (63.6\%) and eight females (36.4\%). Fourteen neonates $(63.6 \%)$ were term, whereas eight (36.4\%) were preterm (Table 1). Eight neonates $(36.4 \%)$ had their first seizures within $24 \mathrm{hrs}$ of birth, while five $(22.7 \%)$ had seizures after $72 \mathrm{hrs}$ of age.

\begin{tabular}{|l|l|l|}
\hline GestationlGender & Male (Percentage) & Female (Percentage) \\
\hline Term & 9 & 5 \\
\hline Pre-term & 5 & 3 \\
\hline Total & $14(63.6 \%)$ & $8(36.4 \%)$ \\
\hline
\end{tabular}

Table 1: Patient demography $(\mathrm{n}=22)$.

The seizure types observed in our series were subtle (17 cases, $77.2 \%$ ), followed by tonic ( 3 cases, $13.7 \%$ ) and clonic ( 2 cases, $9.1 \%$ ) seizures (Table 2). There was no myoclonic seizure in our cases. HIE was the most common etiology ( 13 cases, $59.1 \%$ ) for neonatal seizures, followed by CNS infections (4 cases, $18.2 \%$ ), malformation syndrome ( 2 cases, 9.1\%) and intracranial hemorrhage (2 cases, 9.1\%) (Table 3 ).

\begin{tabular}{|l|l|l|l|}
\hline Type & No. (Percentage) & Pre-term & Term \\
\hline Subtle & $17(77.2 \%)$ & 5 & 12 \\
\hline Tonic & $3(13.7 \%)$ & 1 & 2 \\
\hline Clonic & $2(9.1 \%)$ & 2 & 0 \\
\hline
\end{tabular}

Table 2: Seizure types ${ }^{*}(n=22) .{ }^{*}$ No myoclonic type.

\begin{tabular}{|l|l|}
\hline Location & No. (Percentage) \\
\hline Centrotemporal spike & $7(41.2 \%)$ \\
\hline Frontal spike & $6(35.3 \%)$ \\
\hline Parietal spike & 1 \\
\hline Multifocal spikes & 3 \\
\hline
\end{tabular}

Table 3: Abnormal EEG findings in neonate seizures $(n=17)$.

Acidosis was noted in cases with perinatal asphyxia and shock. Other laboratory data was non-contributory. Neonatal sepsis was diagnosed in $6(27.3 \%)$ cases, with 3 proven bacterial cultures. In the initial EEG, five cases (22.7\%) were normal and seventeen (77.3\%) had abnormal EEG with focal spikes in all. Focal centrotemporal (7 cases, $41.2 \%$ ) or frontal (6 cases, $35.3 \%$ ) spikes were the most common EEG findings. Three $(17.6 \%)$ out of 17 were multifocal spikes. During follow-up, ten (58.8\%) of the 17 cases showed normal EEG 3 months later after diagnosis. Another two cases had multifocal epileptiform discharges, four cases showed focal spikes and one case with slow background in the EEG performed after 3 months old. In our study, nineteen (86.4\%) of 22 cases had good clinical responses after loading with phenobarbital. Phenytoin was added in one case but eventually shifted to levetiracetam due to failure to respond to phenobarbital and phenytoin. The remaining two had no clinical seizure after levetiracetam add-on treatment.

Twelve (54.5\%) cases had abnormal brain sonographic findings including 8 cases of mild to moderate periventricular echogenicity (PVE), 2 severe PVE, 1 multiple intracranial hemorrhage and 1 grade IV intraventricular hemorrhage.

Among 13 cases with HIE, eleven had good neurologic outcomes. One had poor prognosis with cerebral palsy and another one died during follow-up. Among 4 cases with CNS infections, two had good outcome and the others two had poor prognosis. The 2 cases with intracranial hemorrhage had poor outcome. The 3 cases of other etiology showed normal outcome in development (Table 4).

\begin{tabular}{|l|l|l|}
\hline Etiology & No. (Percentage) & Outcome \\
\hline $\begin{array}{l}\text { Hypoxic-ischemic } \\
\text { encephalopathy }\end{array}$ & $13(59.1 \%)$ & $\begin{array}{l}11 \text { good, 1 poor, } 1 \\
\text { expired }\end{array}$ \\
\hline CNS infection & $4(18.2 \%)$ & 2 good, 2 poor \\
\hline Intracranial hemorrhage & $2(9.1 \%)$ & All poor \\
\hline Malformation syndrome & $2(9.1 \%)$ & All good \\
\hline Others & $1(4.5 \%)$ & good \\
\hline
\end{tabular}

Table 4: Etiology and neurodevelopmental outcomes.

\section{Discussion}

In this retrospective study of neonatal seizures, subtle type was the most common in our series. It has been reported to occur in both term and preterm neonates [1]. In our study, neonatal seizures occurred more in male than in female that is compatible with the literature report.

Among the etiologies of neonatal seizures, moderate to severe hypoxic-ischemic encephalopathy is the most important cause of neonatal seizure, followed by CNS infection and metabolic disorders. Our results were similar to other studies [1,2].

Significant abnormal electroencephalographic findings were found in new-borns with perinatal asphyxia, septic shock, meningitis, and ICH and structure abnormality of the brain. EEG abnormalities, such as burst suppression, low voltage, or multifocal discharges are associated with abnormal neurological outcome [3]. Of those who had multifocal spikes or slow background in EEG, as shown in our series, had severe PVE, multiple ICH or grade IV IVH in the initial brain sonography, and developed encephalomalasia on brain MRI follow-up. Therefore, combined multimodality of EEG and neuroimaging data can help us predict the prognosis of patients with neonatal seizure. Contrarily, although aEEG is potentially useful in the NICU, it is less sensitive in the detection of seizures with brief, low amplitude, or epileptic discharge that was distal to the recording site [4-6]. We only use aEEG to the high-risk infants with critical clinical conditions in our NICU.

As for treatment, phenobarbital and phenytoin or fosphenytoin have been reported as the first-line Antiepileptic Drug (AED) in neonatal seizure. Phenobarbital is the preferred drug of choice in treating most neonatal seizures. It is more effective than the unpredictable pharmacokinetics of phenytoin in the neonatal population [7]. The current researches support the safety and efficacy of Levetiracetam 
Citation: Hu SC, Hung KL, Chen HJ (2017) Neonatal Seizures: Incidence, Etiologies, Clinical Features and EEG Findings in the Neonatal Intensive Care Unit. Epilepsy J 3: 117. doi:10.4172/2472-0895.1000117

Page 3 of 3

(LEV) in neonates as the second-line of therapy [8]. Fosphenytoin was not available in this country. Therefore, we chose LEV as the adjunctive treatment to phenobarbital for recurrent or persistent neonatal seizures. Low risk of seizure recurrence after withdrawal of AEDs was observed in 3 out of 58 neonatal seizures in one report [9]. This implicates the goal to minimize unnecessary, long-term use of AED for most neonates with seizures. Our study also demonstrated that the prognosis of neonatal seizures is highly related to the etiology.

In our NICU, all neonates suspected seizure were seen by pediatric neurologists. This study was conducted by pediatric neurologists. A recent report stressed the importance of the involvement of a neonatal neurologist in the multi-disciplinary team approach to provide focused neurologic care to new-borns with neurological diseases such as neonatal seizures [10].

\section{References}

1. Volpe JJ (2001) Neonatal Seizures. In: Volpe JJ (ed.) Neurology of the newborn (4th edn.). Philadelphia: WB Saunders, pp: 178-214

2. http://www.uams.edu/cdh1/default.asp

3. Ortibus EL, Sum JM, Hahn JS (1996) Predictive value of EEG for outcome and epilepsy following EEG confirmed neonatal seizures. Electroencephalogr Clin Neurophys 98: 175-185.
4. Shellhaas RA, Barks AK (2012) Impact of amplitude-integrated electroencephalograms on clinical care for neonates with seizures. Pediatr Neurol 46: 32-35.

5. Shellhaas RA, Soaita AI, Clancy RR (2007) Sensitivity of amplitudeintegrated electroencephalography for neonatal seizure detection. Pediatrics 120: 770-777.

6. Shah DK, Mackay MT, Lavery S, Watson S, Harvey AS, et al. (2008) Accuracy of bedside electroencephalographic monitoring in comparison with simultaneous continuous conventional electroencephalography for seizure detection in term infants. Pediatrics 121: 1146-1154.

7. Painter MJ, Scher MS, Stein AD, Armatti S, Wang Z, et al. (1999) Phenobarbital compared with phenytoin for the treatment of neonatal seizures. N Engl J Med 341: 485-489.

8. Mruk AL, Garlitz KL, Leung NR (2015) Levetiracetam in neonatal seizures: a review. J Pediatr Pharmacol Ther 20: 76-89.

9. Hellstrom-Westas L, Blennow G, Lindroth M, Rosen I, Svenningsen NW (1995) Low risk of seizure recurrence after early withdrawal of antiepileptic treatment in the neonatal period. Arch Dis Child Fetal Neonatal Ed 72: F97-F101.

10. Mulkey SB, Swearingen CJ (2014) Advancing neurologic care in the neonatal intensive care unit with a neonatal neurologist. J Child Neurol 29: 31-35. 